\title{
Health Care Provider Considerations in Providing Care to Aging Patients with Early Life Trauma
}

\author{
Nancy Isserman ${ }^{1^{*}}$ and Bea Hollander-Goldfein ${ }^{2}$ \\ ${ }^{1}$ Operation Home and Healing: Services for Veterans and Families, USA \\ ${ }^{2}$ Transcending Trauma Project, Council for Relationships, USA
}

"Corresponding author: Nancy Isserman, Director of Operation Home and Healing: Services for Veterans and Families, Co-Director of the Transcending Trauma Project, Council for Relationships, Philadelphia, PA 19104, USA, Tel: 2153826680; Fax: 4106057913; E-mail: nisserman@councilforrelationships.org

Rec Date: Oct 07, 2016; Acc Date: Oct 17, 2016; Pub Date: Oct 19, 2016

Copyright: $\odot 2016$ Isserman N, et al. This is an open-access article distributed under the terms of the creative commons attribution license, which permits unrestricted use, distribution, and reproduction in any medium, provided the original author and source are credited.

\section{Short Communication}

Health care providers work with an increasing aging population, one with numerous trauma survivors. The findings of the qualitative research project, the transcending trauma project (TTP) are derived from the analysis of comprehensive interviews of Holocaust survivors and shed light on the aging process impacted by their traumatization during WWII. TTP also interviewed the children of these survivors, who, in the course of their interviews, often revealed their thoughts as primary caregivers of their aging parents. These findings identify key areas that health care providers need to consider when providing treatment to aging patients who have survived trauma earlier in their lives. They also highlight where additional research is needed. Clinicians will benefit from learning how the adult children experience their parents' aging and coping abilities, how they understand their parents' trauma, and how this impacts the roles the adult children play in their aging parents' lives in general and in their parents' medical treatment in specific. TTP research suggests that the roles played by the adult children in their aging parents' medical care impact how the aging parents cope with and comply with medical intervention.

In TTP's database of 96 survivor interviews and 107 children of survivors' interviews, only a small number of children commented on the relationship of the Holocaust and the aging process of their parents. In contrast to the survivor parents, who viewed their own coping strategies as positive responses to difficult circumstances both during the war and afterwards [1], these children viewed the coping strategies of their parents in a negative light. However, this negative perspective on the aging of their Holocaust survivor parents was ameliorated in the children who experienced positive relationships with their parents as they were growing up. The children with positive ties to their survivor parents could, while holding negative views on the aging process, still think of their parents in positive terms. This change did not occur in children who, in addition to holding negative views on the aging process, also evidenced negative relationships with their parents. The contrast between the negative views of aging and the positive views of the relationship with their parents is a finding that needs further exploration. One question to study is how do caretaking roles of aging parents differ between children with positive parental relationships versus children with negative parental relationships? What is the impact of this contrast on the aging parent's behavior? How do these opposing viewpoints affect the treatment and prognosis of the aging parent?

This communication moves beyond the original focus of the TPP on coping and adaptation after extreme trauma. A significant component of TTP's research concentrated on family relationships when questioning the Holocaust survivor family members [2]. A key finding of the project focused on the impact of the Holocaust on the second generation. The quality of the survivors' parenting and the quality of the attachment between the survivors and the second generation mediated the impact of the extreme trauma experiences of the survivor on the second generation. The TTP research included 51 intergenerational families who, after analyzing each generation of interviews within the family, divided into four types of families: positive, negative, mixed, and mediating families. These definitions were derived from the child's descriptions of the following factors: the description of each parent's behavior toward the child and the emotional state of the parent(s); the existence or absence of conflict in the parental marriage; the identification of targeting a child by either parent for which the child experienced a negative impact as a result; the description of the child's adult mental health and relationships; and the existence of empathy in the child toward the war experience of his or her survivor parent(s). Relationships were measured according to the quality of family dynamics paradigm (QFDP), which grew out of TTP's analysis of the relationships between the child and his or her primary caregivers in the family of origin and also out of attachment theory principles. This model incorporates five elements on a continuum. The elements range from close to distant; empathy to selfcenteredness; validation to criticism; positive expressions of feelings to negative expressions of feelings; and communication that is open to communication that is closed [3].

Although not the original focus of the research the TTP interviews also shed light on the aging process, the impact of extreme trauma on aging, and how the relationships between parent and child may affect the caregiver role that the child provides. In several interviews survivors described the dramatic impact of losing their spouses especially in light of the extreme losses experienced in the Holocaust. Even in troubled marriages, the bonds between the partners were strengthened by a mutual understanding of their profound losses during the war and the difficulties they faced in rebuilding their lives in a new country postwar. They remarked in their interviews that the departed spouses were the only ones who comprehended the extent of the trauma that they faced. While they were young and focused on creating a family and building a career they suppressed the devastating memories. The death of the spouse at a time in their lives of diminished activity and health led to a painful awareness of loneliness. At the same time their adult children were focused on creating their own families and careers leaving them less time to help their parents even if the quality of the parent-child relationship was positive. These changing circumstances due to the losses from the Holocaust and now the spouse's death, the diminishing quality of their health, and the changing nature of the parent-child dyad, exposed the survivors to 
unmitigated residual pain from the memories of their Holocaust experiences.

The TTP data analysis also revealed patterns in the parent-child relationships that impacted the children's attitudes as caretakers of their parents. The children who in their descriptions of their relationships with their parents used positive terms such as warm, close, and helpful as adults also expressed positive attitudes towards the parents regardless of how they viewed the aging process. Even when they mentioned that their parents placed emotional demands on them through the parents' depression, decreased affection, anxiety, denial, or lack of communication, the children with childhood positive parent-child ties reported that they could still be empathic, understanding, and grateful for the life lessons that the parents imparted to them. This stands in contrast to the children who experienced negative, troubled relationships with their parents as they were growing up. The children, who saw their parents as distant, selfcentered, and/or angry, did not show empathy towards their aging parents. These children described the aging parents as a burden and an undesirable presence in their lives.

Both the positive and the negative views of aging parents impact the caretaker children's behavior toward their parents. While additional research is needed to discern exactly how these views impact the caretaking role, health care providers need to obtain a clear sense of whether or not the caretaker child is a cooperative partner in the care of the aging parent or an uncooperative partner.

TTP's findings on aging Holocaust survivors and the attitudes of their children towards the aging parents are relevant beyond the Holocaust to the general aging population and specifically, to those who are victims of other traumas. We hypothesize from the TTP analyses of the rich, detailed interviews that the quality of the family relationships acts as a mediator on the aging process of trauma victims, who are vulnerable to the vicissitudes of aging and susceptible to reexperiencing their earlier traumas. We posit that future research will illustrate that positive parent-child relationships positively impact the parent's aging care and treatment and negative parent-child relationships negatively impact the health outcomes. Building on TTP findings if medical practitioners are familiar with the characteristics of the family relationships in the patient's constellation they will better understand their patients' behavior. Understanding the reasons for certain behaviors as well as the strengths and coping abilities of the family especially the family caregivers will facilitate better assessments of the patient's needs and future care. A crucial piece of information in the assessment process of the coping strengths of the patient and the family also comes from the impact of the survivor's early life trauma or traumas on the aging process.

Research is needed to better understand how extreme trauma in early life influences how survivors respond to medical procedures and institutional care. A second factor influencing how survivors respond to medical care is the quality of the relationships with their caregiver children. If it is found that the effectiveness of medical intervention is mediated by the attitude and cooperation of the patient and the family caregivers, then the care of survivor patients would be greatly influenced by their post trauma vulnerability and how their caregivers relate to them. The nature of these relationships, whether positive or negative, could determine the ability of the family system to provide quality support that aids in the survivor's care and/or recovery. Understanding these patterns will lead to medical practitioners who are better equipped to identify gaps in the care and needs of patients who are trauma survivors.

The TTP findings are a result of a secondary analysis of the data from the interviews. They are limited by the fact that parent-child relationships and their influence on aging were not a focus of the original study or interview questions. Thus, further research focused specifically on the impact of the quality of parent-child relationships on the care of aging trauma survivors is needed. A better understanding of the effect of the positive and negative family dynamics on the care of the aging will facilitate better outcomes for aging trauma survivors.

In conclusion, TTP research on intergenerational family dynamics and aging has revealed that children with positive childhood relationships with their parents expressed better attitudes towards providing for the needs of their parents as they encountered the difficulties in the aging process. Health care providers can build on positive parent-child relationships to facilitate positive health care outcomes. Their treatment plans and procedures can be designed to compensate for the deficits in the family relationships that impact negatively on the aging parent's care and prognosis. The treatment plans can also support and strengthen the family relationships that have a positive impact on the parent's care and prognosis thus providing a better outcome in the treatment of the aging trauma survivor.

\section{References}

1. Isserman N, Hollander-Goldfein B, Horwitz SN (2013) In Their Own Words: Survivor Wartime and Late Life Coping Styles. Kavod honoring aging survivors.

2. Hollander-Goldfein B, Isserman N, Goldenberg JE (2012) Transcending Trauma: Survival, Resilience and Clinical Implications in Survivor Families. Routledge, New York, USA.

3. Isserman N (2005) In the National Interest: Defining Rural and Urban Correctly in Research and Public Policy. Int Reg Sci Rev 28: 465-499. 